\title{
Improvement Maneuverability and Stability of Independent 4WD EV by DYC Based on Control Target Dynamic Regulation*
}

\author{
Guangcai ZOU**, Yugong LUO**, Keqiang LI**and Xiaomin LIAN** \\ ${ }^{* *}$ Department of Automotive Engineering, Tsinghua University \\ Haidian Qu, Beijing, P.R.China \\ E-mail:lingboweibu99@mails.tsinghua.edu.cn
}

\begin{abstract}
A direct yaw-moment control (DYC) method based on the dynamic regulation of the control target is proposed to achieve integrated optimization between maneuverability and stability for the independent 4WD EV. Firstly, the yaw rate responses are calculated from the modified bicycle model, which respectively represent the maneuverability and stability of EV. With these responses deduced, the integrated control target for the maneuverability and stability is determined for all steering situations. Furthermore based on the "feedforward+feedback" control structure, the DYC controller is designed which combines the dynamical sliding mode control (DSMC) and LQ control. DSMC avoids the drawbacks of the oscillations by chattering happening in the classical SMC and allows the smoothness of the direct yaw-moment. The simulation experiments show that this DYC system can restrain the side slip angle effectively and keep higher yaw rate, which guarantees the EV maneuverability and stability. Moreover, the robustness of systems for road adhesion conditions variation and vehicle parameters uncertainties is also guaranteed in simulation validation.
\end{abstract}

Key words: Direct Yaw-Moment Control, Independent 4WD, Maneuverability, Stability, Electric Vehicle

\section{Introduction}

In the research of the direct yaw-moment control (DYC) system for the independent 4WD EV, the advantages of independent 4WD and motor drives are combined. The motor torque responds quickly and accurately, and can be measured easily, while the driving force can be distributed arbitrary for all wheels. This combination can improve the EV maneuverability and stability greatly.

On one side, some researchers set the control target as the zero side slip angle response for EV safety to design the DYC system based on LQ method [1 4]. Although the side slip angle is reduced near zero, the steady gain of yaw rate is relative small, which increases the turning radius greatly, i.e. worse maneuverability. The robustness of LQ method against the time-varying parameters is a noticeable problem for these DYC systems in practice, too. On the other side, some different control targets of yaw rate response for EV maneuverability are also utilized for DYC system [5 6], which show some difficulties to restrain the side slip angle for EV safety. For the DYC controller, in [7 9], the classical SMC method is applied in EV DYC system for improving the system robustness. The strong yaw rate and

${ }^{\star}$ Received 19 Nov., 2007 (No. 07-0735) [DOI: 10.1299/jmtl.1.305] 
the direct yaw-moment oscillations by the control chattering is still a problem to solve.

In order to integrated optimize the maneuverability and stability for independent 4WD $\mathrm{EV}$, i.e. to reduce the side slip angle and keep high yaw rate steady gain at the same time, a DYC method based on the dynamic regulation of the control target is proposed in this paper. Firstly, using the road adhesion information from observation and the front wheel steering angle from measurement, the bicycle model is so modified that it can describe the vehicle dynamics in a much larger range. Then based on this improved bicycle model, the yaw rate responses are calculated from the modified bicycle model respectively to represent the maneuverability and stability of EV. The DYC control target is deduced based on these two yaw rate responses with the fuzzy method, which shows the integration of the maneuverability and stability. In order to realize this target yaw rate, the "feedforward+feedback" control structure is utilized for the DYC controller frame. Furthermore the dynamical sliding mode method (DSMC) and LQ method are combined to keep the system robustness and reduce the oscillations of the vehicle motion states in the feedback control process. In the end the required traction/braking torques of all the wheels are distributed under the symmetry strategy, which won't deteriorate longitudinal dynamic performance. The simulation results of the EV motion control by DYC show that this DYC method can integrate control the EV maneuverability and stability by the regulated control target. The robustness of systems for road adhesion conditions variation and system parameter uncertainties is also guaranteed..

\section{Control Target Dynamic Regulation for EV DYC}

According to the " $\beta-\gamma$ " phase plane which describe the vehicle stability, under a certain yaw rate $\gamma$, the side slip angle $\beta$ must be limited in a special range, otherwise the vehicle will lose its stability. [10 11] But if $\beta$ is reduced too much, $\gamma$ will drop greatly. [1 2] The lower $\gamma$ results in the larger turning radius under the constant EV longitudinal velocity, which deteriorates the EV maneuverability. For the balance and optimization between the maneuverability and stability, a new way is proposed in this paper to regulate the dynamic motion target for DYC control. In the aspect of the theoretical analysis for the vehicle motion, the " $\beta-\gamma$ " phase plane can be a good choose for this control target regulation, but this phase plane changes a lot with the variations of the front and rear tire side slip characteristics, the road adhesion condition and the vehicle velocity. The calculation process strongly depends on the dynamical changing of the tire characteristics and is too complex to be applied in real time control. So it is necessary to find another method to represent the vehicle motion state for the maneuverability and stability. In this aspect, the linear bicycle model has been widely utilized, which is relative compact. Although its validity has been proved very effective in many vehicle dynamics control researches, the bicycle model only can be applied in a limited range, i.e. the motion characteristics below the lateral acceleration about $0.4 \mathrm{~g}$. So some modifications are done to improve this linear model and the yaw rate responses are calculated from this modified model respectively to represent the maneuverability and stability of EV. Then the dynamic control target is determined based on these two yaw rate responses. After that the DYC controller is designed to achieve this dynamic target. The whole DYC control structure in this paper is described as in Fig.1, in which the place of this control target regulation part in the DYC system is shown. 


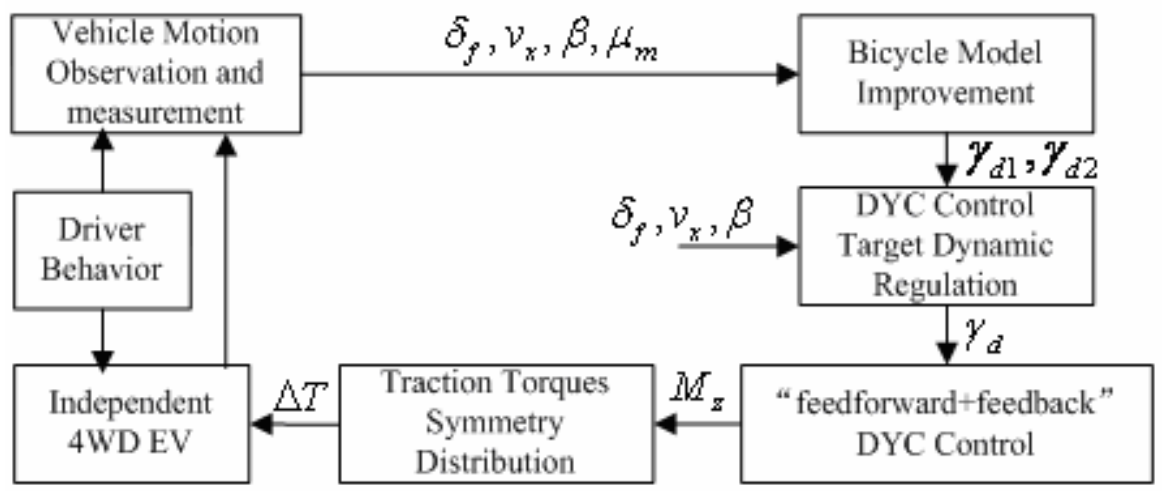

Fig. 1 DYC System for Independent 4WD EV

In terms of the maximum road adhesion factor $\mu_{m 0}$, the nominal cornering stiffness $C_{f 0}$ and $C_{r 0}$ of the front and rear tires used in normal bicycle model can be obtained through the tire experiment. The assumption is assumed that the tire nominal cornering stiffness is proportional to maximum road adhesion factor. So the nominal cornering stiffness $C_{f}$ and $C_{r}$ for front and rear tire with maximum road adhesion factor $\mu_{m}$ can be calculated as in (1).

$$
C_{f}=\frac{\mu_{m}}{\mu_{m 0}} C_{f 0}, C_{r}=\frac{\mu_{m}}{\mu_{m 0}} C_{r 0}
$$

In this paper, the cornering stiffness is determined not using the slope of the tangent though the origin on the tire side slip map but the slope of an appropriate secant.

Since some techniques have been developed to estimate $\mu_{m}$ using the driving wheel with motor drives [11], the bicycle model can be modified to the 3DOF model as shown in (2), which includes the longitudinal acceleration dynamics.

$$
\dot{x}=A x+B u+E \delta_{f}
$$

Where, $x=\left[\begin{array}{ll}x_{1} & x_{2}\end{array}\right]^{T}=\left[\begin{array}{ll}\beta & \gamma\end{array}\right]^{T}, u=M_{z}$,

$$
B=\left[\begin{array}{ll}
0 & b_{2}
\end{array}\right]^{T}=\left[\begin{array}{ll}
0 & 1 / I_{z}
\end{array}\right]^{T},
$$

$E=\left[\begin{array}{ll}e_{1} & e_{2}\end{array}\right]^{T}=\left[\begin{array}{ll}2 \mu_{m} C_{f 0} / \mu_{m 0} m v_{x} & 2 \mu_{m} C_{f 0} l_{f} / \mu_{m 0} I_{z}\end{array}\right]^{T}$,

$A=\left[\begin{array}{ll}a_{11} & a_{12} \\ a_{21} & a_{22}\end{array}\right]=\left[\begin{array}{cc}\frac{-2 \mu_{m}\left(C_{f 0}+C_{r 0}\right)}{\mu_{m 0} m v_{x}}-\frac{\dot{v}_{x}}{v_{x}} & -1-\frac{2 \mu_{m}\left(C_{f 0} l_{f}-C_{r 0} l_{r}\right)}{\mu_{m 0} m v_{x}^{2}} \\ \frac{-2 \mu_{m}\left(C_{f 0} l_{f}-C_{r 0} l_{r}\right)}{\mu_{m 0} I_{z}} & \frac{-2 \mu_{m}\left(C_{f 0} l_{f}^{2}+C_{r 0} l_{r}^{2}\right)}{\mu_{m 0} I_{z} v_{x}}\end{array}\right]$.

$\delta_{f}$ denotes the front wheel steering angle, $M_{z}$ the direct yaw moment, $l_{f}$ and $l_{r}$ the nominal distances from the center of gravity to the front and rear wheel axle respectively, $I_{z}$ the nominal yaw moment of inertia of the vehicle, $m$ the nominal vehicle mass, $v_{x}$ the vehicle longitudinal velocity.

For the control target dynamic regulation of DYC, as the first step, the steady value of the control target is considered. The transfer function from $\delta_{f}$ and $M_{z}$ to $\gamma$ is obtained with (2) by Lap lace transform as in (3).

$$
\gamma(s)=\frac{\left(e_{2} s+e_{1} a_{21}-e_{2} a_{11}\right) \delta_{f}(s)+b_{2}\left(s-a_{11}\right) M_{z}(s)}{s^{2}-\left(a_{11}+a_{22}\right) s+a_{11} a_{22}-a_{21} a_{12}}
$$

On one side, the yaw rate response which represents the maneuverability can be deduced with (3). In this situation, $M_{z}$ is set as zero, while the vehicle steers freely. The steady value 
of $\gamma$ is as in (4).

$$
\gamma_{1}=\frac{\mu_{m}^{2} C_{f 0} C_{r 0} l v_{x}}{\mu_{m 0}^{2} C_{f 0} C_{r 0} l^{2}+\mu_{m 0} \mu_{m} m v_{x}^{2}\left(C_{r 0} l_{r}-C_{f 0} l_{f}\right)} \delta_{f}
$$

Generally speaking the bicycle model is an ideal model for the real vehicle with time-invariant parameters such as the tire side slip characteristic. When the vehicle runs in the nonlinear range of the tire characteristic, the yaw rate response will deteriorate strongly In fact. So $\gamma_{1}$ obtained in (4) can be used to represent the ideal maneuverability of the real vehicle, which also is used as the control target for ESP research on normal road [12]. This relative yaw rate can guarantee the understeering tendency with a positive stability factor.

On the other side, the stability zone in the " $\dot{\beta}-\beta$ " phase plane for the vehicle motion can be simplified to the zone described by (5). [13]

$$
\left|c_{1} \beta+c_{2} \dot{\beta}\right|<1
$$

Despite the variations of the phase plane in different situations, if $\dot{\beta}=\beta=0$, (5) is always correct. So still from (3), $M_{z}$ is applied to keep $\beta$ as zero; the yaw rate response obtained can be used to represent the stability. The steady value of $\gamma$ is as in (6),

$$
\gamma_{2}=\frac{2 \mu_{m} C_{f 0} v_{x}}{-\mu_{m 0} m v_{x}^{2}-2 \mu_{m}\left(C_{f 0} l_{f}-C_{r 0} l_{r}\right)} \delta_{f}
$$

For the integrated optimization of the maneuverability and stability, the steady value of the control target should be a tradeoff between $\gamma_{1}$ and $\gamma_{2}$.

For the dynamic value of the control target, the tire characteristic and the dynamic response characteristic of vehicle steering are considered.

As mentioned above, the cornering stiffness using here is set as the slope of an appropriate secant. So with the tire side slip characteristic, when $\delta_{f}$ keeps small, the front wheel side slip angle is relative small also, which makes the real front wheel cornering stiffness is much higher than $C_{f 0}$. When $\delta_{f}$ increases a lot, the front wheel side slip angle increases also, which makes the real front wheel cornering stiffness is much lower than $C_{f f}$. From (3) and (4), the change of $C_{f 0}$ results in the change of the real yaw rate responses, which are very different from $\gamma_{1}$ and $\gamma_{2}$. In order to correct the yaw rate responses, the linear decreasing function $p\left(\delta_{f}\right)$ is utilized as in (7). Maybe when the nonlinear or more complex function is used, the correction effect will be better. But for the linear function, the coefficients are relatively easy to tune here.

$$
p\left(\delta_{f}\right)=p_{1} \delta_{f}+p_{2}
$$

Where, $p_{1}$ and $p_{2}$ are constants, $p_{1}<0, p_{2}>0$.

For considering the dynamic response characteristic of vehicle steering, as shown in (3), it is can be simplified as a second-order transfer characteristics in the bicycle model. In order to improve the steering response speed of the vehicle, a faster second-order transfer characteristics is utilized to replace the original one.

So the improved yaw rate responses $\gamma_{d 1}$ and $\gamma_{d 2}$ are shown as in (8).

$$
\begin{aligned}
& \tau_{s}^{2} \ddot{\gamma}_{d 1}+2 \tau_{s} \dot{\gamma}_{d 1}+\gamma_{d 1}=p\left(\delta_{f}\right) \gamma_{1} \\
& \tau_{s}^{2} \ddot{\gamma}_{d 2}+2 \tau_{s} \dot{\gamma}_{d 2}+\gamma_{d 2}=p\left(\delta_{f}\right) \gamma_{2}
\end{aligned}
$$

Where, $\tau_{s}$ is the time constant, which is smaller than the corresponding value in (2) by the coefficient $h>1$, which can increase the response speed of the vehicle steering.

$$
\tau_{s}=\frac{1}{h \sqrt{a_{11} a_{22}-a_{12} a_{21}}}
$$

In order to integrated coordinate the maneuverability and stability, the tradeoff must be 
done to combine the two yaw rate responses $\gamma_{1}$ and $\gamma_{2}$. Due to the nonlinear tire characteristic, the analytical solution is difficult to obtain. But the rules to compromise $\gamma_{1}$ and $\gamma_{2}$ can be summarized as follows.

1. When $\beta$ is small, i.e. the vehicle stability is good enough, the vehicle maneuverability should be a priority;

2. When $\beta$ is larger, the vehicle stability should be mainly guaranteed;

3. When $\beta$ is too large to be controlled by the driver himself, the whole control target is to keep the vehicle stability.

Based on the rules above, the fuzzy function $k(\beta)$ can be designed to make the tradeoff for the control target dynamic regulation. The target yaw rate $\gamma_{d}$ is determined as in (10).

$$
\gamma_{d}(s)=[1-k(\beta)] \gamma_{d 1}(s)+k(\beta) \gamma_{d 2}(s)
$$

In this paper, as the same as [12], $2^{\circ}$ is considered to be the maximum limit $\beta_{0}$ which can be controlled by the driver himself. Then $\left|\beta / 2^{\circ}\right|$ is taken as the input of $k(\beta)$. The membership function and the fuzzy logic rules are shown respectively in Fig. 2 and TABLE I. The DYC controller as follows is designed to achieve for the independent 4WD EV. To realize the control target, in theory, the upper limit of the output range for $k(\beta)$ should be one. But according to the calculation process of the fuzzy logic method, if the upper limit of the output range is set as one, the real output will approach one but not reach one. This result can not satisfy the requirement for the "vehicle stability control", because one should be reached in control process when it is necessary. In order to overcome this practical problem from fuzzy logic, the output range is enlarged a little bit so that $k(\beta)$ can reach one really when it is necessary. And after the fuzzy process, $k(\beta)$ is restrained not to exceed one in vehicle control practice.
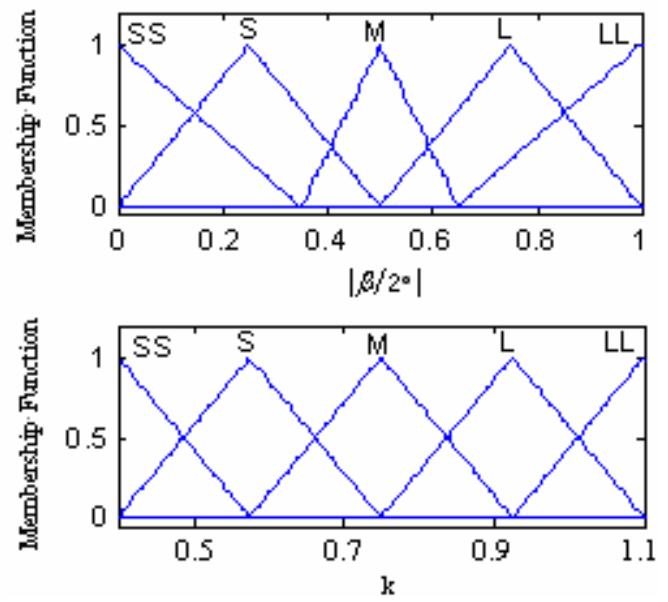

Fig. 2 Membership function for $k\left(\beta, \beta_{0}\right)$

TABLE I FUZZY LOGIC RULES FOR $k\left(\beta, \beta_{0}\right)$

\begin{tabular}{llllll}
\hline \hline$k\left(\beta, \beta_{0}\right)$ & $\mathrm{SS}$ & $\mathrm{S}$ & $\mathrm{M}$ & $\mathrm{L}$ & $\mathrm{LL}$ \\
$\left|\beta / \beta_{0}\right|$ & $\mathrm{SS}$ & $\mathrm{S}$ & $\mathrm{M}$ & $\mathrm{L}$ & $\mathrm{LL}$ \\
\hline \hline
\end{tabular}

When this control target dynamic regulation method is applied, the advantages which can be achieved are:

1. The tendency that the vehicle motion becomes unstable is reduced by restraining the vehicle side slip angle.

2. Integrated coordination of the vehicle stability and the vehicle handling is achieved, which limit the side slip angle in the driver-controllable range and improves the yaw rate response Characteristics. 
3. The smooth transition is realized for the control target changing which is from main handling to complete stability. The riding comfort when the target changes are guaranteed.

4. The final control target is only set as the target yaw rate. So the design of the DYC controller is simpler to a certain extent.

\section{3. "Feedforward+Feedback" DYC Controller Design}

The "feedforward+feedback" control structure is utilized for the DYC controller designed in this paper for the independent 4WD EV. Fig. 3 shows the whole control structure.

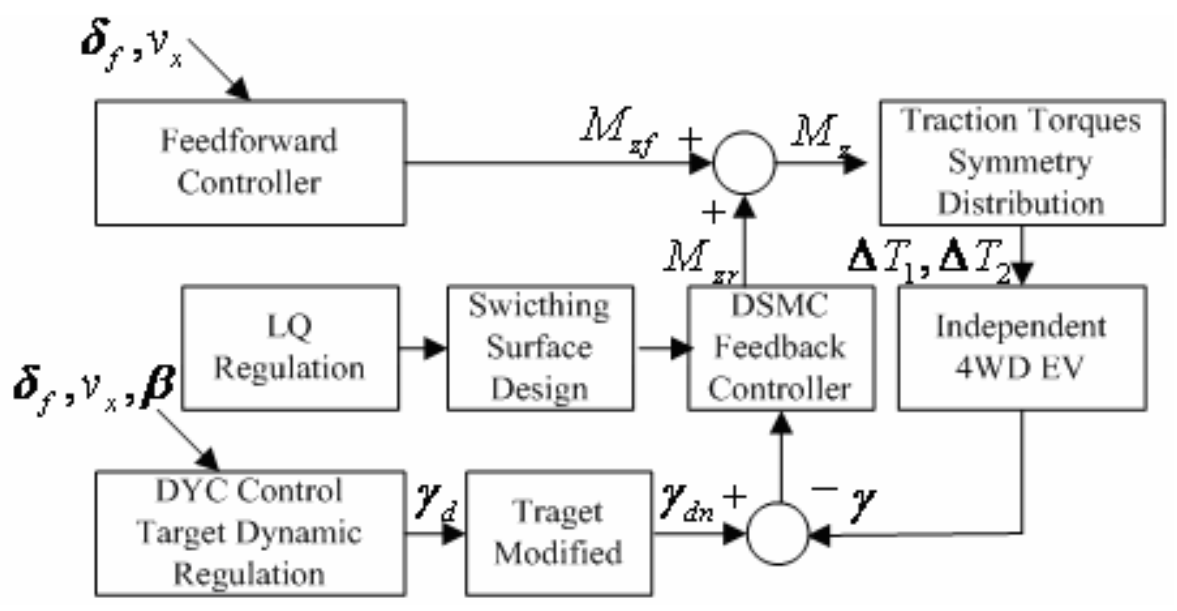

Fig. 3 DYC controller for independent 4WD EV

In this structure, $M_{z}$ consists of two parts, the direct yaw moment $M_{z f}$ of the feedforward controller and the direct yaw moment $M_{z r}$ of the feedback controller. Then $M_{z}$ is realized by the traction torques of all the wheels distributed under the symmetry strategy.

\section{A. Design of Feedforward Compensation}

The feedforward compensation is designed to make the steady value of yaw rate $\gamma$ equal to the target yaw rate $\gamma_{d}$. For this purpose, the relationship between the control input $M_{z}$ and the $\delta_{f}$ is assumed to be as follows:

$$
M_{z f}(s)=G_{f 1} \delta_{f}(s)+G_{f 2} \gamma_{d}(s)
$$

Where, $G_{f 1}$ and $G_{f 2}$ are the proportional gain of the feedforward controller. From (3) and (11), the following equation can be obtained.

$$
M_{f f}=\frac{1}{a_{11} b_{2}}\left(e_{1} a_{21}-e_{2} a_{11}\right) \delta_{f}+\frac{1}{a_{11} b_{2}}\left(a_{21} a_{12}-a_{11} a_{22}\right) \gamma_{d}
$$

So

$$
\begin{gathered}
G_{f 1}=\frac{e_{1} a_{21}-e_{2} a_{11}}{a_{11} b_{2}} \\
G_{f 2}=\frac{a_{21} a_{12}-a_{11} a_{22}}{a_{11} b_{2}}
\end{gathered}
$$

\section{B. Design of Feedback Compensation}

The feedback compensation is designed based on the dynamical sliding mode control (DSMC). In DSMC, the control input $u$ is introduced into the switching surface and $\dot{u}$ is determined. The switching part with the high frequency exists in $\dot{u}$, so $u$ is essentially 
continuous by integral, through which the control chattering occurring in classical SMC is reduced.

The control error is defined as $\Delta \gamma=\gamma-\gamma_{d}$. From (2), the follows equation is obtained.

$$
\dot{z}=A_{r} z+B_{r} u_{r}+F
$$

Where,

$$
\begin{aligned}
& z=\left[\begin{array}{ll}
z_{1} & z_{2}
\end{array}\right]^{T}=\left[\begin{array}{ll}
\Delta \gamma & \Delta \dot{\gamma}
\end{array}\right]^{T}, \quad u_{r}(s)=\left(s-a_{11}\right) M_{2 r}(s), \\
& B_{r}=\left[\begin{array}{ll}
0 & b_{2}
\end{array}\right]^{T}, \quad A_{r}=\left[\begin{array}{cc}
0 & 1 \\
a_{12} a_{21}-a_{11} a_{22} & a_{11}+a_{22}
\end{array}\right], \\
& F=\left[\begin{array}{ll}
0 & f
\end{array}\right]^{T}=\left[\begin{array}{ll}
0 & \frac{e_{1} a_{21}}{a_{11}} \dot{\delta}_{f}-\ddot{\gamma}_{d}+\left(a_{11}+\frac{a_{21} a_{12}}{a_{11}}\right) \dot{\gamma}_{d}
\end{array}\right]^{T}
\end{aligned}
$$

The switching surface for DSMC is

$$
s_{0}=c_{1} z_{1}+c_{2} z_{2}+u
$$

Where, $c_{1}$ and $c_{2}$ are positive and undetermined. Then the reaching law for DSMC is

$$
\dot{s}_{0}=-\varepsilon \operatorname{sgn}(s)
$$

To eliminating the influence from the disturb part $f$ in the control system, $\varepsilon$ is defined as in (18).

$$
\varepsilon=\left|c_{2} f\right|
$$

With $u_{r}(s)=\left(s-a_{11}\right) M_{z r}(s)$ in (15), the feedback compensation is

$$
\ddot{M}_{z r}=a_{11} \dot{M}_{z r}-c_{1} \dot{z}_{1 n}-c_{2} \dot{z}_{2 n}-\varepsilon \operatorname{sgn}(s)
$$

As shown as in (19), the switching part with the high frequency exists only in the second-order differential coefficient of $M_{z}$. By integral $M_{z}$ is smooth enough, which reduce the oscillations by control chattering remarkably and improve the riding comfort when DYC works.

When the control system becomes steady and enters into the sliding mode stage, i.e. $s_{0}=0, \dot{\delta}_{f}=0, \dot{\gamma}_{d}=0$. From (16), the steady control error $\Delta \gamma_{0}$ is proportional to the steady feedback direct yaw moment $M_{z r 0}$ as shown in (20), which is determined by the form of the DSMC switching surface.

$$
\Delta \gamma_{0}=\frac{a_{11}}{c_{1}} M_{z r 0}
$$

In order to reduce the steady error in DSMC, a target modifying method is utilized here based on (20). The target modified is

$$
\gamma_{d n}=\gamma_{d}-\frac{a_{11}}{c_{1}} M_{z r 0}+p_{d}\left(\gamma_{d}-\gamma\right)
$$

Where, $p_{d}$ is a positive constant. The new error is defined as $\Delta \gamma_{n}=\gamma-\gamma_{d n}$, and $z_{n}=\left[\begin{array}{ll}z_{1 n} & z_{2 n}\end{array}\right]^{T}=\left[\begin{array}{ll}\Delta \gamma_{n} & \Delta \dot{\gamma}_{n}\end{array}\right]^{T}$. The control system is transformed as in (22)

$$
\dot{z}_{n}=A_{r} z_{n}+B_{r} u_{r}+F
$$

The new switching surface for DSMC is

$$
s_{1}=c_{1} z_{1 n}+c_{2} z_{2 n}+u
$$

So the system function in the sliding mode stage is 


$$
\left\{\begin{array}{l}
\dot{z}=A_{r} z+B_{r} u_{e q} \\
u_{e q}=-c_{1} z_{1 n}-c_{2} z_{2 n}
\end{array}\right.
$$

Then LQ method is utilized to designed $c_{1}$ and $c_{2}$. The cost function $\mathrm{J}$ is shown in (25).

$$
J=\int_{t_{0}}^{\infty}\left(z_{n}^{T} Q z_{n}+u_{e q}^{T} R u_{e q}\right) d t=\int_{t_{0}}^{\infty}\left(q_{1} z_{1 n}^{2}+q_{2} z_{2 n}^{2}+r u_{e q}^{2}\right) d t
$$

Where, $Q$ and $R$ are both positively definite matrixes. $q_{1}, q_{2}$ and $r$ are all positive. The result for this LQ method is

$$
u_{e q}=-R^{-1} B_{r}^{T} \bar{K} z
$$

Where, $\bar{K}=\left[\begin{array}{ll}k_{11} & k_{12} \\ k_{12} & k_{22}\end{array}\right]$ is the unique positive solution of the Ricatti function shown in (27).

$$
K A_{r}+A_{r}^{T} K+Q-K B_{r} R^{-1} B_{r}^{T} K=0
$$

From (26), $c_{1}$ and $c_{2}$ are

$$
c_{1}=\frac{b_{2} k_{12}}{r}, \quad c_{2}=\frac{b_{2} k_{22}}{r}
$$

In order to keep the longitudinal dynamics performance when the DYC controller works, the equal traction torques is implemented on the two wheels on the same axle but in opposite direction, which is shown as in Fig. 4. In this distribution way, the resultant longitudinal driving force by DYC is zero, so the longitudinal velocity won't be influenced. Moreover this distribution method is relative simple and adapts to work in real time.

$$
\Delta T_{1}=\frac{r_{\omega} l_{r} M_{z}}{2 l_{d} l}, \Delta T_{2}=\frac{r_{\omega} l_{f}}{2 l_{d} l} M_{z}
$$

Where, $r_{\omega}$ is the wheel radius, and $l_{d}$ is the half of the track.

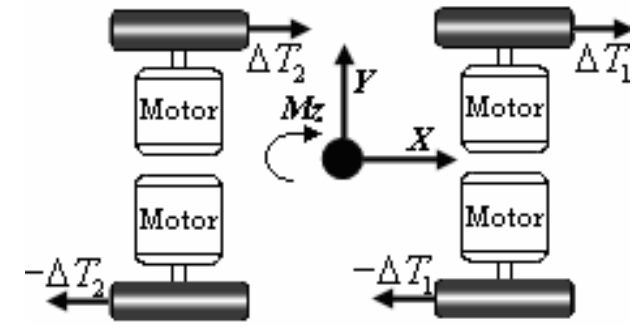

Fig. 4 Symmetry distribution strategy for all wheels torques

\section{Simulation and Analysis}

\section{Vehicle Mode and Tire Model for Simulation}

The similar vehicle model as in [2] is used for the simulation, which contains the longitudinal, lateral and yaw motion of the EV body, the rotation and translation of all four independent wheels.

Assuming that the dynamic property of motor can be expressed as general first-order delay system as in (30).

$$
T(s)=\frac{1}{1+\tau_{m} s} T_{r}(s)
$$

Where, $T$ denotes the real motor torque output, $T_{r}$ the motor torque demand, $\tau_{m}$ the time 
constant of the first-order delay system.

Fig. 5 shows the utilized tire Characteristic under the static wheel load in this model. Assuming that tire force production can be expressed as general first-order delay system as in (31).

$$
F_{x}=\frac{1}{1+\tau_{x} S} F_{x_{-} r e q}, F_{y}=\frac{1}{1+\tau_{y} S} F_{y_{-} r e q}
$$

Where, $F_{x}$ and $F_{y}$ respectively denote the dynamic longitudinal tire force and lateral force, $F_{x_{-} \text {req }}$ and $F_{y_{-} \text {req }}$ respectively the steady longitudinal tire force and lateral force, $\tau_{x}$ and $\tau_{y}$ the time constant of the first-order delay system.

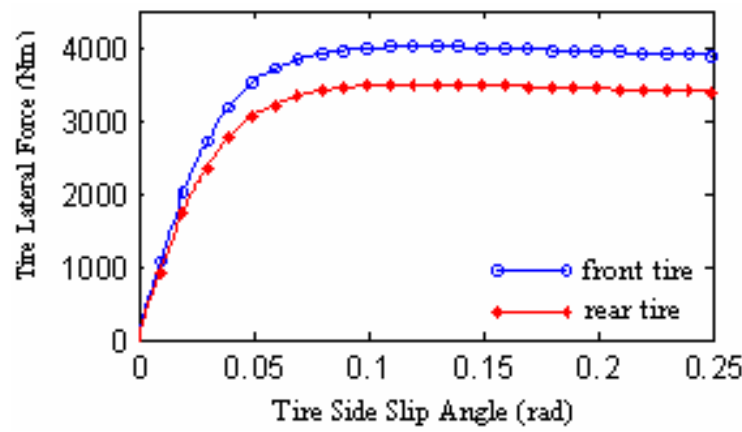

Fig. 5 Tire characteristics under static wheel load $\left(\mu_{m}=0.8\right)$

The main parameters used in the simulation model are shown as in TABLE II.

TABLE II PARAMETERS IN SimUlation MODEL

\begin{tabular}{|c|c|c|}
\hline Parameters & Unit & Value \\
\hline Vehicle Mass $m$ & $\mathrm{~kg}$ & 1460 \\
\hline Vehicle Yaw Inertia $I_{z}$ & $\mathrm{~kg} \cdot \mathrm{m}^{2}$ & 2355 \\
\hline Height of C.G. $h$ & $m$ & 0.50 \\
\hline Distance from C.G. to Front/Rear Axle $l_{f} / l_{r}$ & $m$ & $1.15 / 1.35$ \\
\hline Motor Delay Time Constant $\tau_{m}$ & $S$ & 0.05 \\
\hline Nominal Front Tire Cornering Stiffness $C_{f 0}$ & $\operatorname{Nrad}^{-1}$ & 16780 \\
\hline Nominal Rear Tire Cornering Stiffness $C_{r 0}$ & $N_{r a d^{-1}}$ & 22659 \\
\hline Tire Longitudinal Force Delay Time Constant $\tau_{x}$ & $s$ & 0.021 \\
\hline Tire Longitudinal Force Delay Time Constant $\tau_{y}$ & $s$ & 0.018 \\
\hline
\end{tabular}

\section{Ramp Front Wheel Steering Angle Simulation}

In this simulation, on the road respectively with $\mu_{m} 0.8$ and $\mu_{m} 0.5$, after the vehicle runs straightly at constant speed of $80 \mathrm{~km} / \mathrm{h}$ for 2 second, the front wheel steering angle changes as shown in Fig. 6, which the maximum steering angle is 0.07 rad. The simulation results are shown in Fig. 7 and Fig. 8. 


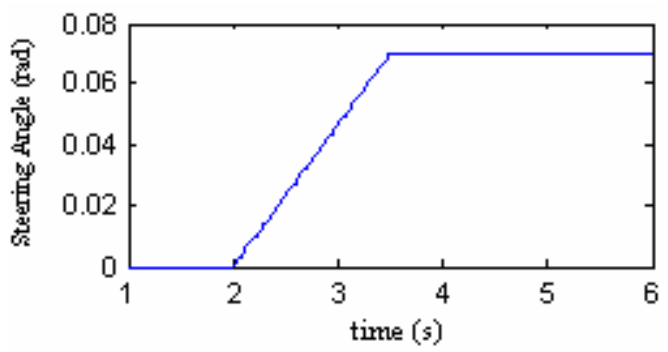

Fig. 6 Ramp front wheel steering angle
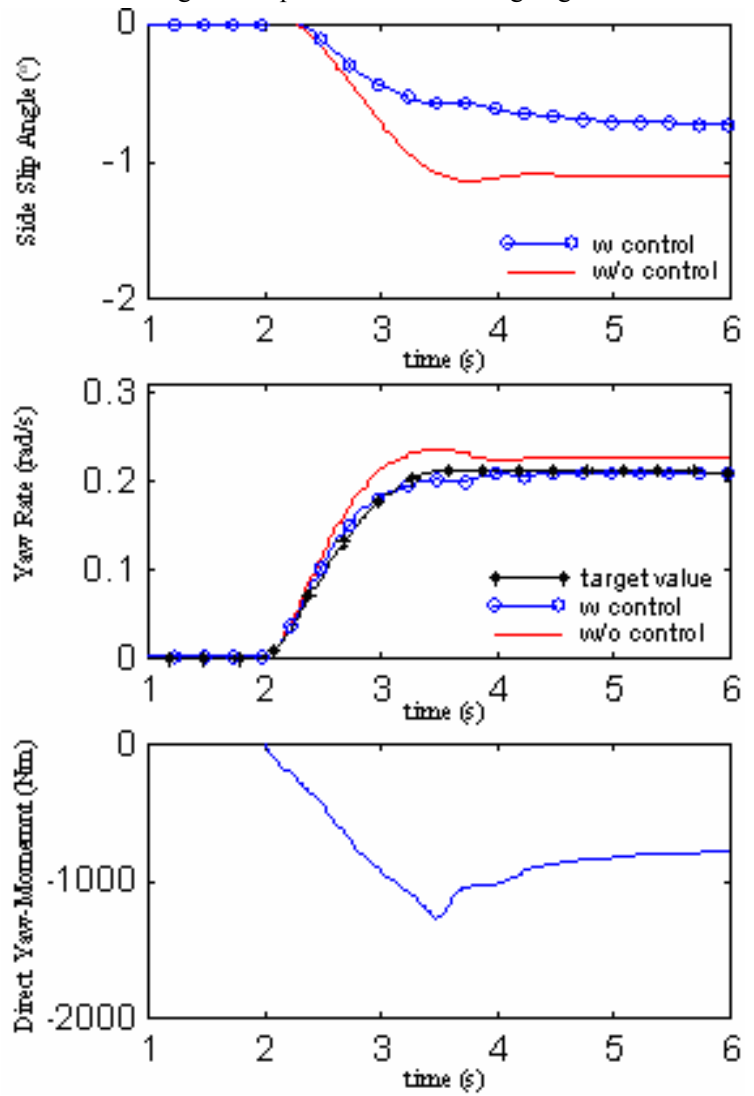

Fig. 7 Simulation results for ramp front wheel steering angle ( $\left.\mu_{m}=0.8\right)$

Fig.7 shows the control results on the road with $\mu_{m}$ 0.8. Compared with the EV without control, the steady value of the target yaw rate with dynamic regulation decreases only about $7.7 \%$. Controlled under this target yaw rate, the side slip angle decreases about $34 \%$, i.e. the turning radius increases only $8.3 \%$. This result shows that the vehicle stability is improved and simultaneously the vehicle maneuverability is kept very well, which proves the validity of this control target dynamic regulation method.

In Fig. 7, under the DYC controller designed in this paper, the yaw rate responds smooth and quickly. With the target modifying method, the steady error is limited below $0.5 \%$, which means higher control precision. The vehicle motion's oscillations due to the control chattering are avoided by the DSMC method, which often occurs in the classical SMC. The direct yaw moment also changes smoothly. 

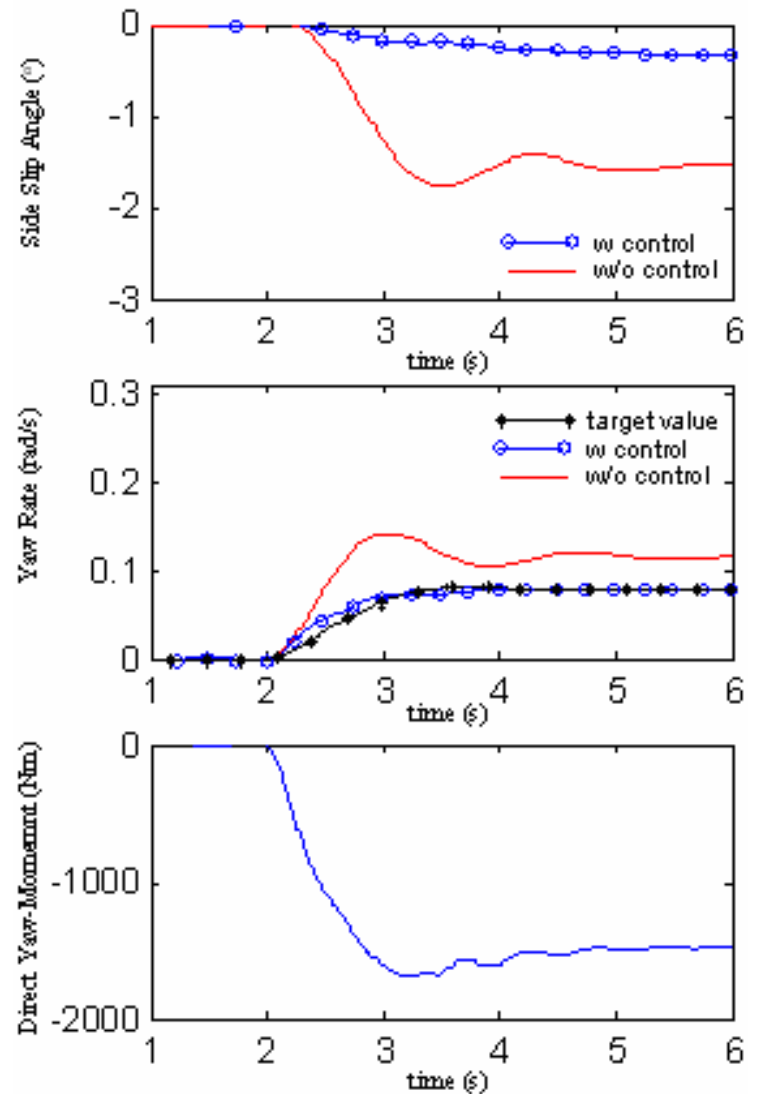

Fig. 8 Simulation results for ramp front wheel steering angle $\left(\mu_{m}=0.5\right)$

Fig. 8 shows the control results on the road with $\mu_{m}$ 0.5. In this situation, the cornering stiffness has dropped greatly. With the front wheel steering angle increasing, the tire lateral characteristic becomes nonlinear obviously. With the DYC method here, the side slip angle decreases about $78.9 \%$, while the yaw rate only decreases $31.6 \%$. By this result, the DYC method proposed in this paper shows good robustness for road adhesion conditions variation.

On the other side, the nominal parameters utilized in the controller are kept unchanged, while the corresponding value in the vehicle model changes as: $m=1750 \mathrm{~kg}$, $l_{f}=1.25 \mathrm{~m}, l_{r}=1.25 \mathrm{~m}, \tau_{m}=0.08 \mathrm{~s}, I_{z}=2800 \mathrm{kgm}^{2}$. The same simulation is done on the road with $\mu_{m}$ 0.8. The result is shown in Fig. 9. 

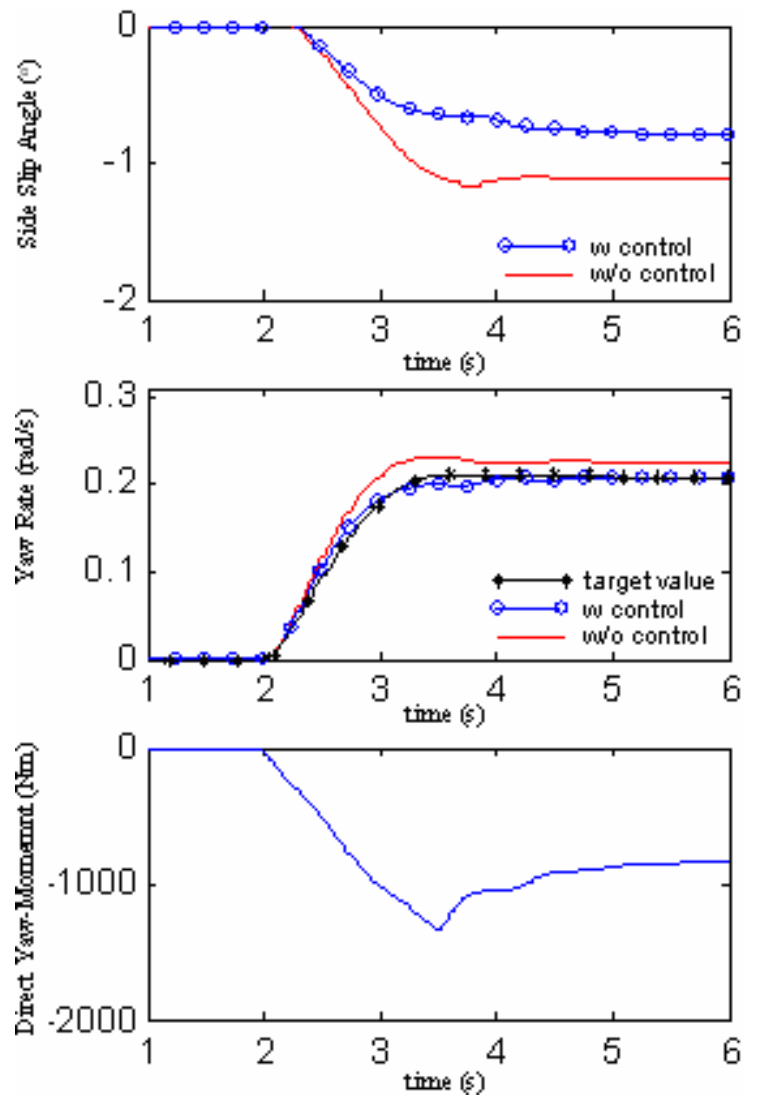

Fig. 9 Simulation results for parameters variation $\left(\mu_{m}=0.8\right)$

In Fig. 9, with the DYC method proposed in this paper, the side slip angle is restrained and the yaw rate is kept well. The former drops about $29.4 \%$, while the latter only drops about $7.3 \%$, i.e. the turning radius increases only $7.9 \%$. The vehicle motion's oscillations are avoided, too. The direct yaw-moment changes still smoothly enough. The result in Fig. 9 shows the same characteristics as in Fig. 7. By this result, the DYC method shows good robustness for the vehicle parameters uncertainties.

\section{E. Sinusoidal Front Wheel Steering Angle Simulation}

In this simulation, on the road respectively with $\mu_{m} 0.8$ and $\mu_{m} 0.5$, after the vehicle runs straightly at constant speed of $80 \mathrm{~km} / \mathrm{h}$ for 2 second, the front wheel steering angle changes as a sinusoidal wave shown as in Fig. 10, which the maximum steering angle is $0.08 \mathrm{rad}$. The simulation results are shown in Fig. 11 and Fig. 12.

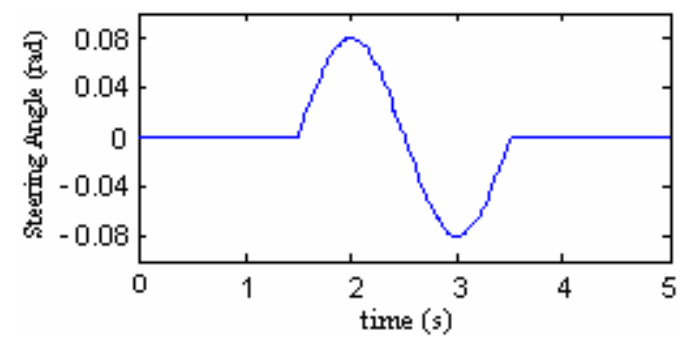

Fig. 10 Sinusoidal front wheel steering angle 

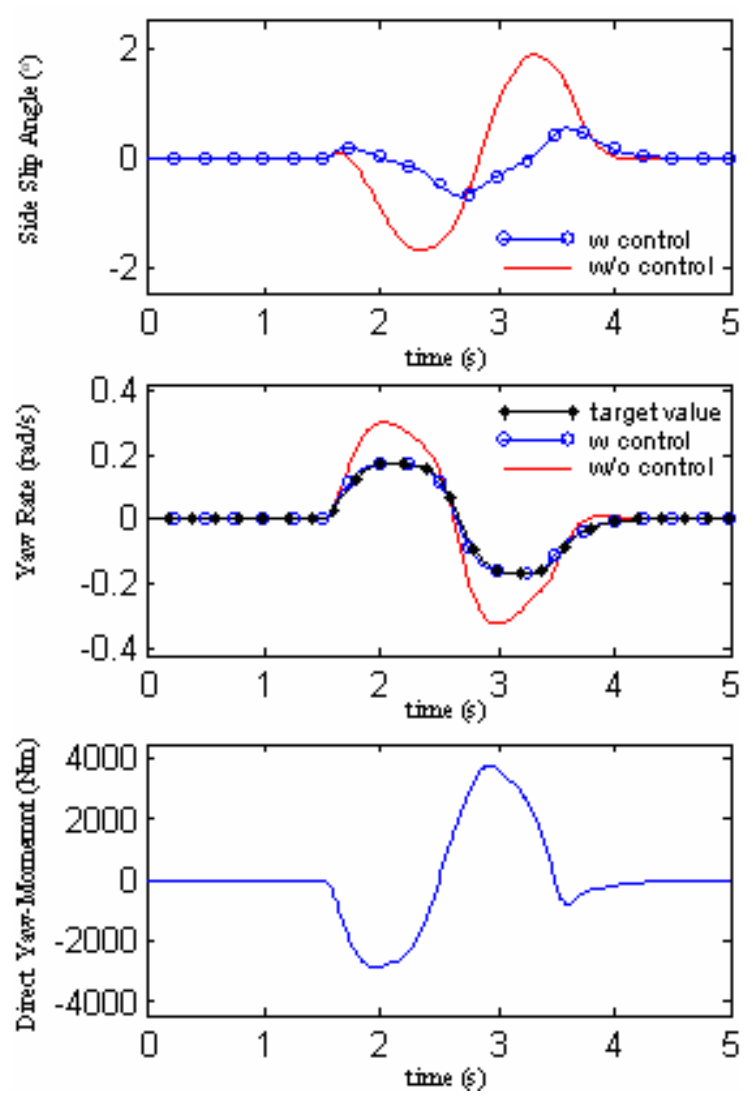

Fig. 11 Simulation results for sinusoidal front wheel steering angle $\left(\mu_{m}=0.8\right)$
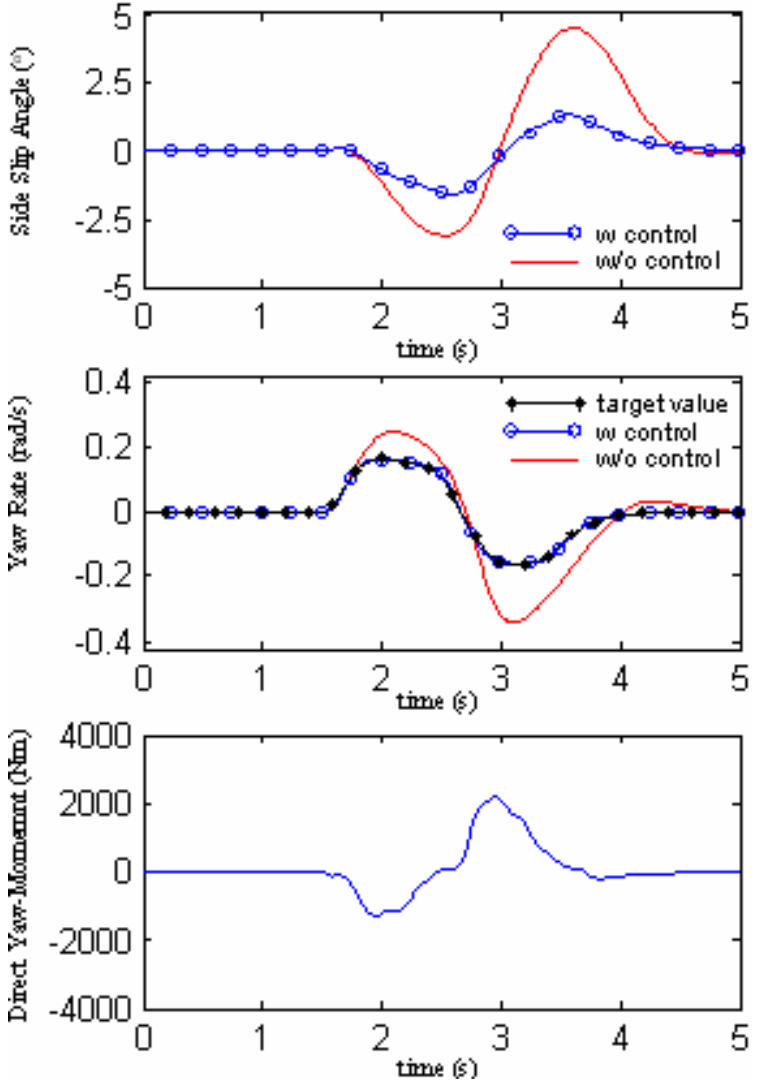

Fig. 12 Simulation results for sinusoidal front wheel steering angle $\left(\mu_{m}=0.5\right)$

The results in Fig. 11 and Fig. 12 show the same characteristic as in Fig. 7 and Fig. 8. With the DYC method proposed in this paper, the EV stability is improved and simultaneously the vehicle maneuverability is kept very well. The vehicle motion's 
oscillations are also avoided on two different roads.

\section{Simulation and Analysis}

A DYC method based on the control target dynamic regulated is proposed for the independent 4WD EV. The research results show:

1) The control target dynamic regulation method for DYC system can restrain the tendency that $\mathrm{EV}$ becomes unstable. The stability and the handling are coordinated to obtain the integrated optimization of the vehicle motion.

2) The control target regulated changes smoothly, which guarantee the riding comfort under vehicle dynamics control.

3) With the DYC controller designed with the combination of DSMC and LQ method, the yaw rate follows its target quickly, accurately and smoothly. This method also shows good robustness for road adhesion conditions variation and the vehicle parameters uncertainties.

\section{References}

[1] Motoki Shino, Masao Nagai, "Independent wheel torque control of small-scale electric vehicle for handling and stability improvement", presented at the JSAE Review 24, 2003: pp449-456

[2] Motoki Shino, Masao Nagai, "Yaw-moment control of electric vehicle for improving handling and stability", presented at the JSAE Review 22, 2001: pp473-480

[3] Peng He, Yoichi Hori, et.al., "Future Motion Control to be Realized by In-wheel Motoroed Electric Vehicle", presented at the Industrial Electronics Society, 2005. IECON 2005. 32nd Annual Conference of IEEE, 2005: pp954-962

[4] Luo Chang. "Implicit Model Following Control for Multi-motor Elect ric Vehicle as a Dynamics System", presented at the China Automotive Engineering, 2005,Vol.27(2): pp164-167

[5] PENG HE, YOICHI HORI, "Improvement of EV Maneuverability and Safety by Disturbance Observer Based Dynamic Force Distribution”, presented at the EVS22, 2006: pp1818-1827

[6] Makoto Kamachi, Kevin Walters, "A Research of Direct Yaw-Moment Control on Slippery Road for In-Wheel Motor Vehicle", presented at the EVS22, 2006: pp2122-2133

[7] Huang Zhi, Zhong Zhihua, "A Study on Active Handling Stability Cont rol for Electric Vehicle with Individual Wheel Driving", presented at the China Automotive Engineering, 2005, Vol.27(5): pp565-569

[8] Remus Pusca, Youcef Ait-Amirat, et. al. "Fuzzy logic based control for electric vehicle with four separate traction drives", presented at the IEEE Vehicular Technology Conference Proceedings 2002. pp.2089-2096

[9] Remus Pusca, Youcef Ait-Amirat, et. al. "Modeling and Simulation of a Traction Control Algorithm for an Electric Vehicle with Four Separate Wheel Drives", presented at the IEEE Vehicular Technology Conference 2002. pp1671-1675

[10] Ken'uchi KITAHAMA. “Analysis of Vehicles' Handling Behavior using a Phase Plane”, presented at the AVEC02.

[11] Shoji Inagaki, Ikuo Kshiro, et.al. "Analysis on Vehicle Stability in Critical Cornering Using Phase-Plane Method", presented at the AVEC94.

[12] Anton T.van Zanten, Rainer Erhardt, et. al. "VDC system development and perspective". SAE Paper 980235.

[13] Ken Koibuchi, Mosaki Yamaoto, et. al. "Vehicle Stability Control in Limit Cornering by Active Brake", SAE paper 950759.

[14] Kimihisa Furukawa, Yoichi Hori, "Recent Development of Road Condition Estimation Techniques for Electric Vehicle and their Experimental Evaluation using the Test EV “UOT March I and 11"', presented at the 29th Annual Conference of the IEEE. 2003. Vol.1:pp925-930 